\title{
Screening des abdominellen Aortenaneurysmas mit Ultraschall - Zahlen, Daten, Fakten
}

Ultrasound Screening in Abdominal Aortic Aneurysm - Numbers, Data, Facts

Autoren

Institute
A. Hyhlik-Dürr ${ }^{1}$, S. Debus ${ }^{2}$, H.-H. Eckstein ${ }^{3}$, W. Lang ${ }^{4}$, T. Schmitz-Rixen ${ }^{5}$, D. Böckler ${ }^{1}$

Die Institutsangaben sind am Ende des Beitrags gelistet.
Schlüsselwörter

- Aneurysma

D Aortenaneurysma

- Screening

- Sonografie

rupturiertes Bauchaortenaneurysma

\section{Key words}

D aneurysm

aortic aneurysm

D screening

o ultrasound

ruptured abdominal aortic aneurysm

\section{Erratum}

A. Hyhlik-Dürr, S. Debus, H.-H. Eckstein, W. Lang, T. Schmitz-Rixen, D. Böckler

Screening des abdominellen Aortenaneurysmas mit Ultraschall - Zahlen, Daten, Fakten

Zentralbl Chir 2010; 135: 403-408

Der Artikel enthält einen Fehler in der Schreibweise eines der Autoren. Der Name des einen Autors muss D. Böckler lauten, nicht D. Boeckler.

Außerdem muss der englische Titel korrekt lauten: Ultrasound Screening in Abdominal Aortic Aneurysm - Numbers, Data, Facts.

\section{Bibliografie}

DOI http://dx.doi.org/

10.1055/s-0031-1283720

Zentralbl Chir 2011; 136: 390

(c) Georg Thieme Verlag KG

Stuttgart · New York .

ISSN 0044-409X

\section{Korrespondenzadresse}

Alexander Hyhlik-Dürr

Universität Heidelberg

Gefäßchirurgie

Im Neuenheimer Feld 110

69120 Heidelberg

Deutschland

Tel.: 06221/5638049

Fax: $06221 / 565423$

ahduerr@web.de 